\title{
Temperature Drift Compensation of Eddy Current Sensor under High Temperature Environment
}

\author{
Ping $\mathrm{He}^{1}$, YunKai $\mathrm{Ma}^{2}$, and Hui $\mathrm{Chen}^{3}$ \\ ${ }^{1}$ HIT,hepinghit@126.com, Harbin, Heilongjiang, China \\ ${ }^{2}$ HIT, 1127345182@qq.com,Harbin,Heilongjiang, China \\ ${ }^{3}$ SACTI, 201109, Shanghai, Shanghai, China
}

\begin{abstract}
Eddy current sensor is an sensor based on eddy current effect. In practical engineering applications, the ambient temperature of eddy current sensor may be up to $135{ }^{\circ} \mathrm{C}$. The temperature drift of eddy current sensor magnifies the error of displacement detection. In this paper, the main factors that cause temperature drift are analyzed in detail, and the results show that the compensation based on single parameter can not meet the demand of high-precision measurement. For this reason, this paper proposes an external compensation method which applies mathematical fitting to realize compensation for temperature drift. The experimental results show that the measurement accuracy of the external compensation method reaches $0.25 \%$ in the working temperature range, which greatly improves the measurement accuracy of eddy current sensor under high temperature.
\end{abstract}

\section{Introduction}

The eddy current sensor is a special inductance sensor that is based on the eddy current effect [1]. Eddy current sensor has many advantages, such as high sensitivity, fast dynamic response and non-contact detection, which makes the sensor widely be used in displacement measurement, speed measurement, temperature measurement, vibration measurement, thickness measurement, eddy current testing(ET) and so on. However, eddy current sensors are very susceptible to temperature changes,especially in high temperature environment. The measurement error, which can't be ignored in the high temperature area, makes the use of the eddy current sensor be limited in the high temperature environment. Analysis shows that temperature affects almost all the materials of the sensor system, so it is difficult to achieve a good effect by traditional single parameter compensation. In this paper, the influence of temperature is estimated by the method of mathematical fitting. Experiment proves that the external compensation method may achieve high precision.

\section{Working Principle}

When the metal panel is in a changing magnetic field, the induced electromotive force inside the metal panel will generate an electric current.The current is closed inside the metal panel and become an eddy current [2]. As shown in figure $1, \mathrm{X}$ is the distance between the coil and the metal conductor,the eddy current density changes as the $\mathrm{X}$ changes, and the intensity of the eddy current varies with the variation of the distance $\mathrm{X}$.

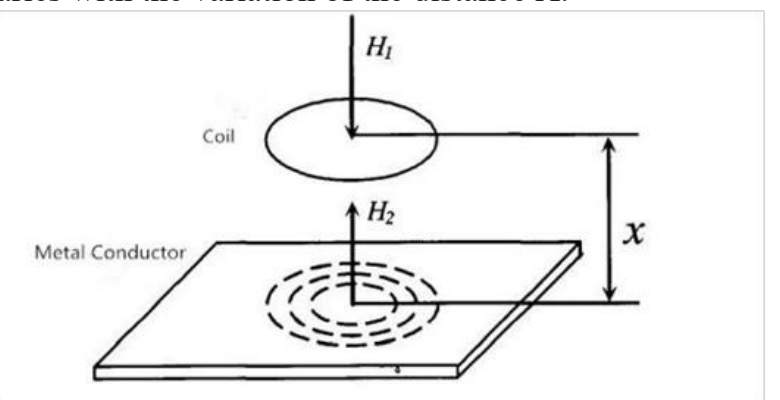

Fig. 1. Principle of Eddy Current Effect

The change of eddy current will cause the change of coil impedance. The factors related to the coil impedance change are: resistivity $\rho$ of the tested material, the distance $\mathrm{X}$ between the coil and the metal conductor, the permeability $\mu$ of the metal conductor, and the frequency $\mathrm{f}$ of the coil excitation current $\mathrm{f}$. To control factors $\rho, \mu, \omega$ do not change, the impedance of the coil is only related to the distance $\mathrm{X}$. We need to measure the distance, that is to say, if we kept $\rho, \mu, \omega$ constant, and the impedance $\mathrm{Z}$ is only a function of distance $\mathrm{X}$ as

$$
Z=f(X)
$$

The eddy current displacement sensor can be made as long as the suitable probe coil and circuit parameters are selected [3]. The structure of the eddy current sensor is simple, mainly composed of the probe, the extended cable and the proximitor. The above three are combined with the tested mechanism to form a basic working system. Working principle diagram of eddy current displacement sensor as shown in Figure 2 . 


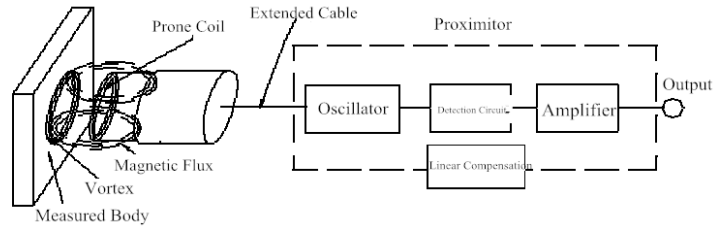

Fig. 2. Basic Principle of Eddy Current DisplacementSensor

The high frequency oscillating current in the preamplifier flows into the coil in the probe through an extended cable and produces alternating electromagnetic fields in the coil of the probe. If there is no metal material around the magnetic field, the magnetic field energy is actual total loss; when the metal material near the magnetic field,the induced current is produced on the metal surface. At the same time, the current field also produces an alternating magnetic field that is opposite to the direction of the head coil.Due to the reaction of magnetic field, the amplitude and phase of the high frequency current of the probe coil has changed .This change is related to the permeability and the conductivity of a metal conductor, the shape and the size of the coil, the frequency of the current and the distance from the probe coil to the metal material and so on. We maintain a constant in all conditions except the distance from the probe metal material, the impedance $Z$ of the coil and the distance $\mathrm{X}$ have a function relation. Through the proximitor, the change of the distance between the probe and the metal becomes a change in the output of the voltage. The eddy current sensor is based on this principle to measure the displacement of metal materials.

\section{Compensation for Tempereature Drift}

The output voltage of the eddy current sensor is affected with temperature drift.So some scholars apply temperature compensation for the eddy current sensor. A common method is to use multi-strand lines to reduce the influence of temperature on the coil impedance by reducing the $\mathrm{AC}$ resistance of the coil, other methods include optimizing the parameters of the sensor, so as to improve the sensor's ability of restraining temperature drift. These methods have achieved effective results, but the factors affecting the temperature drift is varied, the single parameter compensation can only meet the general requirements. If the project requires high precision, external compensation method that uses mathematical fitting will achieve comprehensive compensation for temperature drift.

In order to determine the effect of high temperature on the sensor, the displacement sensor is installed on the displacement calibration table which is in the incubator. Adjust the distance of the target with the center point of the sensor. The temperature of the incubator is set at room temperature of $20^{\circ} \mathrm{C}$, and change the temperature according to $20^{\circ} \mathrm{Cinterval}$ as a measurement point. Then change the displacement between the sensor and the target, 21 displacement points are measured at each temperature, the interval between every two displacement points is $0.1 \mathrm{~mm}$, read the sensor output voltage of each measurement point, output voltage data of eddy current sensor as shown in table land table 2 .
Table 1. Output Voltage of Eddy Current Sensor 1

\begin{tabular}{|c|c|c|c|}
\hline $\begin{array}{c}\text { Displacement } \\
(1 / 10 \mathrm{~mm})\end{array}$ & $\begin{array}{l}20^{\circ} \mathrm{C} \\
\text { (v) }\end{array}$ & $\begin{array}{l}40^{\circ} \mathrm{C} \\
\text { (v) }\end{array}$ & $\begin{array}{l}60^{\circ} \mathrm{C} \\
\text { (v) }\end{array}$ \\
\hline 1 & -9.989 & -10.054 & -10.096 \\
\hline 2 & -8.997 & -9.051 & -9.21 \\
\hline 3 & -8.004 & -8.049 & -8.213 \\
\hline 4 & -7.015 & -7.044 & -7.212 \\
\hline 5 & -6.024 & -6.049 & -6.193 \\
\hline 6 & -5.031 & -5.041 & -5.169 \\
\hline 7 & -4.068 & -4.016 & -4.134 \\
\hline 8 & -3.071 & -2.998 & -3.093 \\
\hline 9 & -2.086 & -1.962 & -2.057 \\
\hline 1 & -1.091 & -0.928 & -0.983 \\
\hline 11 & -0.094 & 0.131 & 0.074 \\
\hline 12 & 0.91 & 1.176 & 1.152 \\
\hline 13 & 1.915 & 2.226 & 2.225 \\
\hline 14 & 2.93 & 3.294 & 3.33 \\
\hline 15 & 3.94 & 4.36 & 4.415 \\
\hline 16 & 4.94 & 5.423 & 5.553 \\
\hline 17 & 5.96 & 6.493 & 6.613 \\
\hline 18 & 6.961 & 7.575 & 7.755 \\
\hline 19 & 7.968 & 8.665 & 8.9 \\
\hline 20 & 8.968 & 9.77 & 10.067 \\
\hline 21 & 9.989 & 10.137 & 10.137 \\
\hline
\end{tabular}

Table 2. Output Voltage of Eddy Current Sensor 2

\begin{tabular}{|c|c|c|c|c|}
\hline $\begin{array}{c}\text { Displacement } \\
(1 / 10 \mathrm{~mm})\end{array}$ & $\begin{array}{l}80^{\circ} \mathrm{C} \\
(\mathrm{v})\end{array}$ & $\begin{array}{c}100^{\circ} \mathrm{C} \\
(\mathrm{v})\end{array}$ & $\begin{array}{c}120^{\circ} \mathrm{C} \\
(\mathrm{v}) \\
\end{array}$ & $\begin{array}{c}135^{\circ} \mathrm{C} \\
(\mathrm{v}) \\
\end{array}$ \\
\hline 1 & -10.096 & -10.096 & -10.096 & -10.096 \\
\hline 2 & -9.512 & -9.761 & -10.086 & -10.096 \\
\hline 3 & -8.509 & -8.76 & -9.123 & -9.437 \\
\hline 4 & -7.498 & -7.805 & -8.152 & -8.493 \\
\hline 5 & -6.483 & -6.804 & -7.177 & -7.532 \\
\hline 6 & -5.46 & -5.801 & $\begin{array}{l}-6.194 \\
\end{array}$ & -6.583 \\
\hline 7 & -4.45 & -4.788 & -5.215 & -5.616 \\
\hline 8 & -3.408 & -3.782 & -4.229 & -4.651 \\
\hline 9 & -2.36 & -2.747 & -3.226 & -3.664 \\
\hline 1 & -1.296 & -1.706 & -2.221 & -2.691 \\
\hline 11 & -0.217 & -0.663 & -1.199 & -1.698 \\
\hline 12 & 0.848 & 0.37 & -0.199 & -0.721 \\
\hline 13 & 1.911 & 1.417 & 0.786 & 0.231 \\
\hline 14 & 2.987 & 2.456 & 1.799 & 1.208 \\
\hline 15 & 4.075 & 3.515 & 2.827 & 2.195 \\
\hline 16 & 5.178 & 4.594 & 3.882 & 3.189 \\
\hline 17 & 6.299 & 5.685 & 4.926 & 4.215 \\
\hline 18 & 7.45 & 6.792 & 5.97 & 5.207 \\
\hline 19 & 8.603 & 7.918 & 7.038 & 6.219 \\
\hline 20 & 9.77 & 9.02 & 8.094 & 7.225 \\
\hline 21 & 10.137 & 10.137 & 9.167 & 8.251 \\
\hline
\end{tabular}

From table 1 and table 2, we can draw the curve of the eddy current sensor output voltage varying with the detection displacement at different temperatures, and draw all the temperature curves on a map for comparison, as shown in figure 3 . 


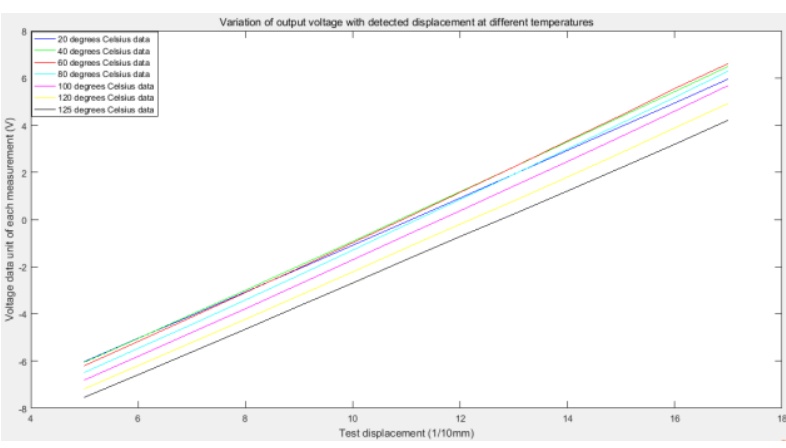

Fig. 3. Variation of output voltage with detected displacement at different temperatures

For 21 data at each temperature, the following seven sets of voltage-displacement equations are obtained by using the MATLAB fitting curve.

$$
U=\left\{\begin{array}{l}
0.99867 * \mathrm{X}-11.047 ; \mathrm{T}=20^{\circ} \mathrm{C} \\
1.04560 * \mathrm{X}-11.325 ; \mathrm{T}=40^{\circ} \mathrm{C} \\
1.06920 * \mathrm{X}-11.610 ; \mathrm{T}=60^{\circ} \mathrm{C} \\
1.06600 * \mathrm{X}-11.888 ; \mathrm{T}=80^{\circ} \mathrm{C} \\
1.04080 * \mathrm{X}-12.066 ; \mathrm{T}=100^{\circ} \mathrm{C} \\
1.00770 * \mathrm{X}-12.259 ; \mathrm{T}=120^{\circ} \mathrm{C} \\
0.97794 * \mathrm{X}-12.451 ; \mathrm{T}=135^{\circ} \mathrm{C}
\end{array}\right.
$$

In the formula (2),

U-sensor output voltage (V);

$\mathrm{X}-10$ times of the measured displacement $(\mathrm{mm})$;

$\mathrm{T}$ - temperature $\left({ }^{\circ} \mathrm{C}\right)$;

From the above seven equations, it can be found that the relationship between the output voltage of the sensor and the measured displacement is highly linear at the same temperature. But the temperature drift of the sensor is quite obvious at different temperatures. If the displacement at high temperature is calculated by the equation at normal temperature,the error is relatively large. So we should seek a better compensation method for temperature drift error under the high temperature environment.

Through the analysis, compared with $20^{\circ} \mathrm{C}$, the output characteristics of the eddy current sensor have changed considerably at $135{ }^{\circ} \mathrm{C}$ high temperature. Take the displacement data of $135^{\circ} \mathrm{C}$ into temperature of $20^{\circ} \mathrm{C}$ voltage-displacement equation, the error voltage is above $1 \mathrm{~V}$, the accuracy of error is above $5 \%$, the traditional method is to compensate for a single parameter or several parameters, and often can only apply to a lower temperature range (less than $100{ }^{\circ} \mathrm{C}$ ). The traditional method for high temperature zone (above $100{ }^{\circ} \mathrm{C}$ ) compensation can not achieve good results. The influence of high temperature on the temperature drift of sensors is critical. So, In this paper, a mathematical fitting method is proposed to get the relationship between all kinds of temperature and the slope and intercept, and the external compensation is done through the formula calculation.

First, fitting the function relation of voltage-displacement straight slope at different temperatures. In order to make the fitting error smaller, higher order fitting is applied,the final fitting result is shown in figure 4 below.

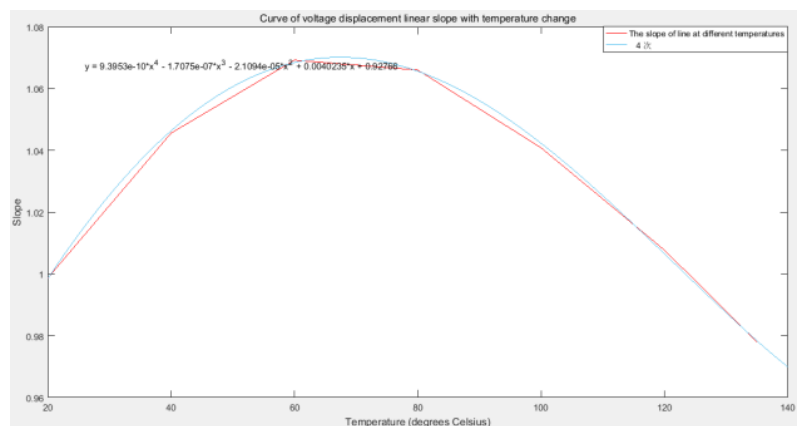

Fig. 4. Curve of voltage displacement linear slope with temperature change

The equation of the relation between the line slope and the temperature is

$\mathrm{K}=9.3953 * 10^{\wedge}(-10)^{*} \mathrm{~T}^{\wedge} 4$

$-1.7075^{*} 10^{\wedge}(-7)^{*} \mathrm{~T}^{\wedge} 3-2.1094 * 10^{\wedge}(-5)^{*} \mathrm{~T}^{\wedge} 2+0.0040235^{*}$

$\mathrm{T}+0.92766$

In the formula(3), $\mathrm{K}$ - line slope;

$\mathrm{T}$ - temperature $\left({ }^{\circ} \mathrm{C}\right)$;

It can be concluded that the influence of temperature on the slope is nonlinear, and gradually increases with the increase of temperature, and then gradually decreases with the increase of temperature.

Then fitting the relationship between the linear intercept at different temperatures and fitting results as shown in figure 5 below

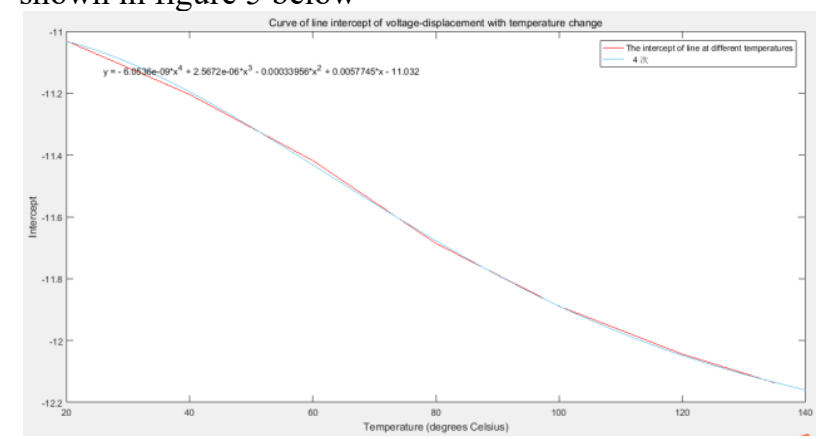

Fig. 5. Curve of line intercept of voltage-displacement with temperature change

The relation equation between the linear intercept and the temperature is

$\mathrm{B}=-1.5984^{*} 10^{\wedge}(-10)^{*} \mathrm{~T}^{\wedge} 4+4.7603^{*} 10^{\wedge}(-10)^{*} \mathrm{~T}^{\wedge} 3$

$-0.00044815^{*} \mathrm{~T}^{\wedge} 2+0.0018916^{*} \mathrm{~T}-10.942$

In the formula (4), B - straight line intercept;

$\mathrm{T}$ - temperature $\left({ }^{\circ} \mathrm{C}\right)$;

It can be seen that the influence of temperature on intercept is almost linear, and the intercept gradually decreases with the increase of temperature. This is because the resistance increases with the increase of temperature, and the output of sensor decreases with the increase of resistance. By fitting the relationship between the function coefficient of the output voltage of the sensor and the temperature, the influence of temperature on the temperature drift of the sensor can be compensated. 
Finally, get the relationship between the output voltage and the displacement of the sensor

$$
\mathrm{U}=\mathrm{K}^{*} \mathrm{X}+\mathrm{B}
$$

In the formula (5),

$\mathrm{K}$ - line slope; B - linear intercept;

$\mathrm{U}$ - sensor output voltage (V);

$\mathrm{X}$ - measurement displacement $* 10(\mathrm{~mm})$.

\section{Conclusion}

First, the error analysis of the external compensation of mathematical fitting is carried out in this paper.

The project required precision is $0.25 \%$, and the sensor output voltage range is $-10 \sim 10 \mathrm{~V}$, so the output voltage error is no more than $0.05 \mathrm{~V}$, after compensation. When the measurement range is less than $1.2 \mathrm{~mm}$, the sensor can meet the precision of $0.25 \%$, reaching the goal of the project. The error under the different temperature of each measurement point within the $1.2 \mathrm{~mm}$ is shown in figure 6 .

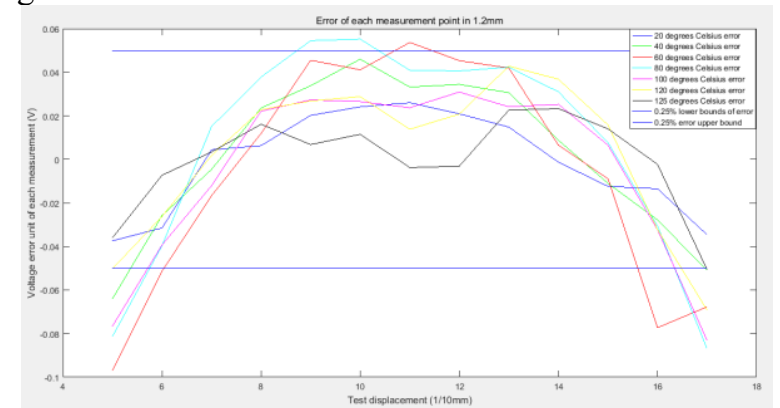

Fig. 5. Error of each measurement point within $1.2 \mathrm{~mm}$

According the above analysis, the main factors affecting the output voltage of electric eddy current sensor temperature drift is the resistance of the test coil, and there are many factors affecting the resistance parameters. Due to its dispersion, the use of conventional compensation that only optimizes the parameters as much as possible to reduce temperature drift can not achieve full compensation. In this paper, we propose to compensate the temperature drift of the sensor by fitting the experimental data. Experiments show that the external compensation can achieve up to $0.25 \%$ accuracy, which is obviously better than the general compensation method for the similar sensor $(2.34 \%)$ [6].

\section{References}

1. Xianjun Wang, Design of differential temperature drift compensation of eddy current sensor and transducer (Zhengzhou University,2006)

2. Baoqiang $\mathrm{Du}$, Comprehensive compensation for temperature drift of eddy current sensor, Semiconductor Technology,50(2004)

3. Qianqian Si,Stability evaluation and experimental study of eddy current sensor,,Beijing University of Chemical Technology,7(2011)

4. Qianqian Si,Coil material of the eddy current sensor temperature drift, Sensor World,26(2011)

5. Qiuhua Fang.Temperature drift compensation of eddy current sensor. Journal of Southeast University, 5(1995)

6. Baoming Ling, Xiangbin Zhuge, Yun Ling, Study on the temperature stability of the eddy current sensor, Eddy Current Sensor 345(1994) 